\title{
Stability challenges not addressed by harmonized guidance - AAPS workshop of the stability focus group, April 3rd- 4th, 2017 in Rockville, MD
}

\author{
Mohd M. Khan ${ }^{1 \dagger}$, Bowen Jiang ${ }^{2 \dagger}$, Anthony Mazzeo ${ }^{3 *}$ and Kim Huynh-Ba ${ }^{4}$
}

\begin{abstract}
An American Association of Pharmaceutical Scientists (AAPS) workshop on stability challenges for clinical supplies and commercial distribution of drug product that are not addressed in the International Conference on Harmonization (ICH) Quality documents was held from April 3rd - 4th, 2017 in Rockville, MD. Seventeen subject matter experts (SME), from industry and the Food \& Drug Administration (FDA) presented and facilitated the round-table discussions. A total of fifty-five participants that included experienced pharmaceutical scientists, both from small and large pharmaceutical companies and service providers, benefited from the opportunity to interact face-to-face with industry partners and regulatory agency SMEs.

The two-day meeting was divided into five major sections to ensure face-to-face interactions and round-table discussions between participants and SMEs: 1) statistical approaches to stability, dissolution, and shelf life testing, 2) microbiological quality of drug products, 3) strategies to support distribution, unplanned excursions, and transportation of drug products, 4) regulatory considerations on stability testing of biologics, and 5) in-use stability during clinical and commercial phases. All in all, this interactive workshop focused on challenges and successes of addressing stability concerns that affect pharmaceutical development, manufacturing, distribution, and use of drug substances/products for which no or limited ICH guidance exists. The interactive meeting provided a unique opportunity to industrial scientists and regulatory agency liaisons to facilitate the discourse on how to address stability challenges that are not addressed in harmonized guidelines: this paper summarizes those discussions.
\end{abstract}

\section{Background}

An AAPS workshop on "Stability Challenges not Addressed by Harmonized Guidance" was held on April 3rd - 4th, 2017 at Hilton Washington, D.C./Rockville Hotel and Executive Meeting Center, Rockville, MD. The meeting was organized by the members of the Stability Focus Group steering committee to facilitate faceto-face discussion with members of regulatory agencies to address stability concerns through science and riskbased approaches. Seventeen subject matter experts (SME), both from industry and the Food \& Drug

\footnotetext{
* Correspondence: tony.mazzeo@bms.com

Mohd M. Khan and Bowen Jiang contributed equally to this work.

${ }^{\dagger}$ Equal contributors

${ }^{3}$ Bristol-Myers Squibb Company, 1 Squibb Drive, New Brunswick, NJ 08903, USA

Full list of author information is available at the end of the article
}

Administration (FDA), presented and facilitated the round-table discussions.

During drug development and commercial distribution of drug products, there are a number of regulatory expectations on their stability for which there is limited guidance from the International Conference on Harmonization (ICH). These expectations have been learned through experience and interactions with various health authorities. Examples where the guidance is limited include a) the impact of temperature excursions during distribution, b) in-use stability once a product is opened from multiuse packaging or when constituted after distribution, c) photostability requirements for light-sensitive products, especially during in-use conditions, d) holding and storage of bulk drug substance and drug product, e) freeze/thaw cycle impact on biologics, 
semi-solids, and liquids, f) stability requirements for biologics, and g) microbiological quality assessment of drug products in a post-penetrated container system. To evaluate product stability and ensure quality, it is important to design studies with clear purpose that employ robust technical approaches and end-to-end information. This information is crucial for regulatory agencies to understand that the product is delivered to patients without any changes in safety or efficacy. As this problem relates to real-world development, manufacturing, distribution, life-cycle management, and use of drug substances and products for which no or limited harmonized guidance exists, SME and attendees raised and discussed the following points:

Topic A: Statistical approaches to stability, dissolution, and shelf life testing

1. The pharmaceutic batches are tested postmanufacture process to ensure product quality. The qualified materials are then stored appropriately. Product distribution is also controlled and pharmaceutical companies do evaluate for temperature excursions. Although supply chains are often designed and qualified to minimize temperature excursions for biologics, it is impractical to keep cold chain products consistently at the recommended label storage conditions throughout the course of production, distribution, and consumer use. Most companies include temperature and humidity monitoring devices to ensure that drug products are maintained within the acceptable range during shipping and distribution. In some cases for extremely sensitive products, excursion limits are defined on the Certificate of Analysis or the product label. Hence there should be allowances for sudden unplanned temperature excursions, provided these excursions fall within the Stability Thermal Budget (STB). STB is defined as "the amount of time (e.g. time out of refrigeration (TOR), time at a given temperature) a drug product in the primary container can spend, outside of registered label conditions without risk to patients and product shelf life". In fact, temperature outside of registered label conditions is as crucial as time for the drug stability. Current GMP related expectations of regulatory authorities, and industry alike, especially for biologics, puts additional pressure on the the STB, which may affect the end use of the product. As a consequence, new statistical simulation strategies and tools are required to better evaluate the allowances for a product undergoing unplanned temperature excursions. These statistical tools can also be employed for determining "what if (unplanned/contingency)" scenarios, to facilitate informed specification negotiations. The statistical/ simulation tools that take into consideration different times and temperatures ranges for batch release, assembly, operations, packaging, distribution, shelf life period, and patient-use/in-use period expect to provide flexibility and risk-based business decisions. Such strategies help assure quality product to the patient without undue burden on the commercial supplier.

2. Participants agreed that there is a need of statistical strategies and tools that efficiently determine factors affecting tablet dissolution. The statistical approaches are expected to consider different aspects of dissolution including drug formulation, packaging, processing, storage, dissolution apparatus, and analytical systems thereof in simulation. The statistical approach has to take temperature and relative humidity ranges in consideration for successfully simulating a dissolution profile. One such example of statistical tools is ASAPprime, which was presented (Li et al. 2016) for predicting the stability of active pharmaceutical ingredients (APIs) in solid-dosage forms as well as the slowdown in dissolution of two APIs in an immediate release tablet. ASAPprime based modeling, that uses a relative humidity-modified Arrhenius equation, efficiently determined the effects of temperature ( $\mathrm{T}$ in $\mathrm{K}$ ) and relative humidity ( $\mathrm{RH}$ ) ranges on dissolution for different storage times. As with chemical stability, water activity rather than water content of the tablet was found to be critical, with the overall impact of $\mathrm{T}$ and $\mathrm{RH}$ following a modified Arrhenius behavior.

3. Statistical methods for constrained stability assays were also proposed; a constrained assay has several components whose reported values restrict each other in one way or another (Zhang 2016). In essence, several stability-indicating tests are normalized based on different measurements of a single common chromatographic assay, for example, cation exchange chromatography and size exclusion chromatography. This normalization of tests, in order to reflect proportions of the entire assay peak area, imposes a constraint among all of the individual tests; some of those may not yet be acknowledged in current analytical practice or guidelines. As a result, negative correlations among the test results may lead to inefficient statistical analysis, prediction, and inconsistency in specification setting. To solve this problem, a multivariate methodology that was originally developed in the field of geosciences, was proposed. This framework efficiently accounts for the tests' normalization constraint thereby removing the above discussed constraints and/or negative 
correlations. Two modeling methods, known as Aitchison and Dirichlet (Aitchison 1986; Maier 2014; Muthukumarana and Tiwari 2016), were discussed, and their advantages and limitations were evaluated using anlysis of biologic stability charge variants as a case study.

4. Ever wonder what defines the true product shelf life? In the meeting, statistical approaches to predict the true product shelf life were discussed. When it comes to the drug product expiration date, users, regulators, and the manufacturers have somewhat different expectations. These expectations could be interdependent to each other in certain aspects. For instance, the user expects the drug products to be effective and safe up until the expiration date. The regulatory agency expects that the acceptance criteria are fulfilled for every stability sample and the current regulatory guidance then recommends the expiration date, which is applied to future manufactured lots produced with the same manufacturing process. The current $\mathrm{ICH}$ methodology estimates the product shelf life using a confidence limit (95\%) for the batch(es) to show the longest time duration that still fulfills the acceptance criteria. Unfortunately, the strategy to manage the out-of-sample risk, whether using the current $\mathrm{ICH}$ approach or treating batches as random, when applied to example data sets were not very effective in estimating (true) product shelf-life (i.e., when a particular batch would no longer fulfill acceptance criteria). Hence, alternative strategies to estimate shelflife must accurately balance the risks that are associated with calculating shelf-life. For example, an unacceptable percentage of nonconforming lots/samples are expected if the storage time is too long in contrast to when it is too short, exposing manufacturers to potential loss. A PQRI perspective on 'considerations in the determination of shelf life' focused on alternative advanced statistical methods to estabilish shelf lives (Stroup and Quinlan 2016; PQRI Stability Shelf Life Working Group, 2007). Recent statistical methodologies such as a Bayesian approach, mixed-model intervals, and quantile regression have shown to be effective in deriving the true shelf-life; however, there remains a gap in adapting $\mathrm{ICH}$ guidance to establishing shelf-life (reviewed by Capen et al. 2012).

\section{Topic B: Microbiological quality of drug products}

Some sterile drug products require reconstitution, admixing, dilution, and withdrawing doses before the drug is administrated to the patient. This requires penetration of the container closure system in a hospital pharmacy setting. Although the process should be handled using sterile techniques, there is no way to assure that the container was not contaminated with microorganisms. Since each drug product differs in its ability to support microbial growth, the post-penetration and admixture storage time and temperature that is provided in the drug product label should be product-specific and supported by scientific data.

There are recorded instances of nosocomial infections that occurred because of contaminated multi-dose vial (MDV)/ single-dose vial (SDV)/Admixtures (NoglerSemenitz et al. 2007) following multiple-use, microbial contamination post-preparation (Bennett et al. 1995), and a case study of microbial contamination of MDVs and associated outbreaks from 1983 to 2002 determined 27 different fungi, Gram-negative, Gram-positive, and protozoa (Mattner and Gastmeier 2004). In light of reported outbreaks induced by contaminated MDVs and possible high risk of MDVs contamination in hospital, FDA's Center for Drug Evaluation and Research (CDER) expects the microbiological studies to be provided in NDAs, ANDAs, and BLAs in support of the post penetration storage time labeling information. It is expected that the final product storage periods be based of a "risk assessment report" that details whether the product does (or doesn't) support adventitious microbial contaminants under the storage conditions. To design the risk assessment studies, a few factors should be taken in consideration, such as: 1) suggested challenge microbes could be $\mathrm{USP}<51>$ microbes, typical skin microflora, nosocomial infection microbes, psychrophilic microbes etc., 2) to simulate contamination, small inoculum size is suggested, provided the inoculum size is quantifiable and repeatable. However, in cases where a membrane filtration method is used, $<100 \mathrm{CFU} / \mathrm{mL}$ inoculum size may serve the purpose, 3) storage conditions, for instance storage temperature(s), diluent(s) etc., should simulate the conditions described in the label, 4) periodic sampling times should be performed, making sure to acquire the time points that are at least as long as the requested maximum storage period. All in all, the information for a given drug product should be product-specific, obtained using risk-based studies that are supported by scientific data obtained for the development of product holding periods. It should be noted that the studies for sterile nonpreserved products preparation (reconstitution, admixing and withdrawing doses for later administrations) in the clinical and/or commercial settings should be considered.

\section{Topic C: Strategies to support distribution, unplanned excursions, and transportation of drug products}

1. The ICH guidelines assume that shipping is validated and controlled, as the quality, efficacy, and safety of drugs are, in part, dependent on the transportation conditions (PDA technical report. 53, 2012). 
Regulatory agencies do allow excursions during shipping albeit based upon well-controlled shipping conditions, which in low-cost transport settings are not effectively controlled, particularly in remote areas of the world. During transport, products could be exposed to i) temperature extremes, ii) humidity extremes, iii) high light exposure, iv) accidents on route that lead to changes of shipping route, v) air pressure changes particularly in ships and aircraft, vi) severe agitations/drops/shocks, vii) unclear shipping details etc. In addition to the ICH recommendations, further risk analysis studies on drug stability are needed that can evaluate the impact of severe excursions, as mentioned above, on drug products that may have been exposed to unusual transport conditions or events, particularly for vaccine products. These studies can analyze the stability risks for three major excursion categories, 1) factors that can be controlled but usually are not addressed, for instance high light intensity in certain parts of the world, 2) factors, such as poor transport or handling, that can be controlled, and 3) unexpected excursions, e.g. transport/shipping system breakdown. These kinds of studies will help evaluate and determine safety margins for the drug products, while making sure the drugs are efficiently supplied without increased risk, due to transport conditions, to the patients. An excellent discussion of stability studies for handling and transport conditions of pharmaceutical and biologics as well ICH recommendations are reviewed by Ammann 2011.

2. Drug product manufacturers are required to maintain good storage and distribution practices throughout the supply chain to maintain product quality from manufacture to the patient. To properly maintain and apply GMP and ICH requirements, the cost of proper shipping and transport can become prohibitive. Hence to come-up wit the ICH recommendations $h$ an efficient Quality Management System (QMS), it is recommended that 1) drug product be stored according to label conditions making sure all personnel involved in supply chain responsibly facilitate proper transport, 2) critical factors, such as storage and transport management, that help employ QMS efficiently should be evaluated separately for each transport mode to better understand the risks associated with transport, 3) product-specific storage and transport control documents should be based on stability data, 4) mean kinetic temperature, although used as an approximation of the impact of temperature excursions, may not be appropriate for biologics as it assumes an Arrhenius effect often not seen with such drugs, 5) it is important to work closely with suppliers to establish and control upper and lower temperatures for product storage and transport, and 6) it is crucial to maintain good documentation that details any excursions that happen during storage/transport. Recently, the United States Pharmacopeia (USP)'s Packaging and Distribution Expert Committee added a new general guidance chapter for storage and movement of investigational drug products, including drug device combinations and non-commercial investigational drug products, which is added to the Good Storage and Distribution Practices for Drug Products family of chapters (USP general chapter prospectus, 1079.1, 2016).

3. Current harmonized guidance dictates that the hold times for drug substance intermediates, product intermediates, and bulk product are systematically established, but does not fully address the specific requirements for the bulk hold time stability study. The reason for this lack of flexibility is that the guidance definition of the date of manufacture is "the date when active ingredients are combined with other ingredients" for shelf life derivation. However, alternative methods of shelf life can be derived using "the date of stable intermediate being further processed for downstream process" instead of the date of manufacture. To be able to establish an alternative shelf life, stability studies that can establish sequential bulk hold times for drug substance intermediates, product intermediates, as well as bulk product are required. This can be achieved during the development phase encompassing Phase I-III IND/CTA and NDA/ WMA filing and the derived alternative shelf life methodology made applicable for later bulk manufacturing process.

\section{Topic D: Stability testing of biologics and regulatory considerations}

1. A manufacturing process change could occur both in development and commercial phases, which requires a comparability study to ascertain that the quality, safety and efficacy of drug products are comparable pre-change and post-change.. Stress studies, are commonly performed in exaggerated storage conditions to understand the stability of biologics in a short period of time. For biologics, a change in the manufacturing process may trigger certain stress stability studies to verify if there is any change to the drug degradation profile.

Forced degradation studies are also useful for drug candidate selection, stability indicating method development, formulation and manufacturing process development, and understanding the 
degradation pathways. Factors to consider for forced degradation studies include $\mathrm{pH}$, temperature, light, oxidizing agents, mechanical stress and freeze-thaw, etc. For example, thermal stress studies are generally performed at $10^{\circ}$ to $20^{\circ} \mathrm{C}$ below the melting temperature $\left(\mathrm{T}_{\mathrm{m}}\right)$ of biologics, as suggested by the presenter.

Leveraging existing knowledge, forced degradation studies could also be used to define the Critical Quality Attributes (CQAs). On the other hand, CQAs helps define subsequent forced degradation studies when there is a major change in process or formulation.

Having a good scientific understanding of the product and process can help interpret non-comparable results.

2. Freeze-thaw is a common practice during biologics manufacturing. However, protein stability might be compromised during freezing or the thaw process due to cold denaturation, ice formation and freeze concentration. Formulations should be optimized to include cryoprotectants when increase in aggregation after freeze-thawing is observed. For instance, the critical parameters of a freeze-thaw process could include time, temperature, container geometry, batch size, handling/process and number of cycles.

Freeze-thaw studies are also performed during various stages of drug product development. During the preclinical stage, freeze-thaw studies are performed to identify the development risk with lead candidates and to evaluate if a drug candidate fits to the platform formulations and process of phase 1 . During the early stages of clinical trials, freeze-thaw studies are utilized to verify the platform formulations and process performance to ensure successful manufacturing. In the later stages, lead drug substance and drug product formulations are evaluated for freeze-thaw stability to verify the formulation robustness. In summary, fit-for-purpose freeze-thaw studies should be built into the drug substance and drug product formulation and process development from the very beginning.

3. Many biologics degrade during photostability studies under the ICH light conditions including visible light and UV exposure. However, most biologics drug substance and product are usually manufactured in a visible light environment containing a small portion of UV light. In a photostability study comparing the ICH light conditions with ambient or mild light, all monoclonal antibodies tested were significantly degraded under ICH light conditions, while several of them were stable under ambient light conditions. In conclusion, ambient or mild light photostability may be more relevant to real manufacturing process of drug products than the ICH photostability testing (Sreedhara et al. 2016).

4. What makes a good stability indicating assay? Stability indicating assays need to be sensitive to changes in critical quality attributes. This requires that, when these assays are performed consistently over time, adequate precision and accuracy should be achieved. Although it is desirable to use compendial methods, many stability-indicating methods used to evaluate product stability cannot be harmonized, which could be attributed to product specificity or complexity of drug products. When dealing with non-compendial methods, the critical quality attribute should be identified to select a method, which is sensitive and specific to determine changes in critical quality attributes. The method should be optimized to meet needed precision, accuracy and robustness. To qualify the assay method, validation has to be conducted and an assay control strategy has to be determined by demonstrating that the assay performs according to its intended use. Similar to harmonized methods, performance of system suitability tests should be monitored during a routine use of a non-compendial method.

5. Regulatory agency expectations were discussed. The purpose of stability studies is to ensure the safety and efficacy of product through shelf-life. As required in 21 CFR 211.166, "There shall be a written testing program designed to assess the stability characteristics of drug products. The results of such stability testing shall be used in determining appropriate storage conditions and expiration dates." Both FDA and ICH published a couple of guidances for industry. The types of stability studies includes studies supporting label storage, accelerated stability studies, and stress/forced degradation studies. It is desirable to perform the stress/forced degradation studies during phase 1 and phase 2 , and it is a regulatory expectation that such data will be available during phase 3 trials. It is a common practice to place the toxicology and the first GMP lot on stability studies, to collect the stability data throughout the clinical development to support licensure. To propose the product shelf-life, stability data from at least 3 lots representative of commercial process stored in commercial containers should been collected. The ongoing stability studies should be completed according to the stability protocol post 
approval. The container closure system (CCS) also needs to be approved for compatibility with drug products, with minimal leachable and no impact on to product quality and safety. In-use stability studies support the stability of the product under actual conditions of use. Stability of drug substance, drug product and any process intermediates are expected after shipping in the intended commercial supply chain. Additional stability should be performed for process intermediates during the manufacturing process, raw materials, and reference standards, etc.

\section{Topic E: In-use stability during clinical and commercial phases}

1. The ICH Q1B guideline describes the basic protocol for photostability testing to understand the impact of light on pharmaceutical drug substances and products during manufacturing, packaging, storage and distribution. However, the guideline does not cover photostability during or after administration. This lack of guidance leaves a significant burden for the industry to plan and effectively execute studies that will assure stability and effective use of photosensitive products. Both small molecular drug and biologics will require a certain level of photostability studies

In-use photostability studies are required for topical drug products as they are applied to external body surfaces, thus, they have the potential to be exposed to a significant amount of light during use by the patient. To design an in-use photostability test, information is needed regarding the longest hold time out of package and patient use characteristics after application. In-use photostability testing is also needed for injectable products, since a significant amount of photodegradation can occur in the line between intravenous (IV) bag and the patients (even when the bag itself is protected from light).

2. Investigational drugs, and drugs submitted for registration, may require some preparation before use. These preparations may be in the form of admixture of the drug with vehicles for oral administration or injection. Data to show the preparations are stable after preparation and through any specific hold time in the clinic is required by health authorities. Except for a general guidance to conduct studies for multidose packages (EMA 2001), there is no guidance specifying the amount of data, design of study, or other details pertaining to ensuring stability of prepared admixtures. To design reasonable in-use stability studies for all phases of developments, it is a good practice to mimic the clinical preparation and usage after discussing with clinicians on details and considering the clinical study integrity. It is agreed by the attendees that the formal in-use stability data should be collected before filing IND/IMsPD/CTA and presenting in the submission for either small molemule drug or biologics. The type and amount of data are risk driven. The EMA guidance was discussed but like other guidances there is not much detail and much is left to the investigator company to determine the scope of the study. The data support the product usage guidelines in the clinic, or details a worst-case scenario that brackets the instructions/ conditions in the Investigator Brochure.

3. While there is a regulatory expectation that in-use shelf-life will be determined for multi-dose packaging, there is little definition of what that testing entails. In-use conditions can be from opening of a package or constituting a product. Similarly, scientific justification for acceptability is required when shipping or storage conditions exceed specifications (excursions). The impact in both situations can be effectively modeled using ASAPprime based on accelerated data which provides the needed understanding of the impact of temperature and humidity for a given product (Waterman et al. 2016). A set of reasonable parameters were proposed to define the in-use scenario for solid products (tablets, capsules) in bottles (Waterman 2009).

\section{Overall conclusion}

The meeting was very successful in bringing together regulatory agencies and pharmaceutical industry personnel for a face-to-face interaction to address the challenges that are not addressed in the ICH Quality documents. Round-table discussions facilitated sharing of technical information and common practices among participants; however, for many topics addressed, attendees were not able to reach a consensus viewpoint. The AAPS Stability Focus Group has further plans to continue facilitating the discussion and interactions of subject matter in future meetings.

\footnotetext{
Abbreviations

AAPS: American Association of Pharmaceutical Scientists; APIs: Active Pharmaceutical Ingredients; CCS: Container Closure System; CQAs: Critical Quality Attributes; EMA: European Medicines Agency; FDA: Food \& Drug Administration; ICH: International Conference on Harmonization;

IV: Intravenous; MDV: Multi-dose Vial; PDA: Parenteral Drug Association; QMS: Quality Management System; RH: Relative Humidity; SME: Subject Matter Experts; STB: Stability Thermal Budget; TOR: Time out of Refrigeration; USP: United States Pharmacopeia
}

\section{Acknowledgements}

The authors gratefully acknowledge the support from all the Subject Matter Experts, who presented and participated in the round-table discussion. We would like acknowledge Kenneth C. Waterman (FreeThink Technologies, Inc.), Jianmei Kochling (Sanofi U.S.), Yan Wu (Merck \& Co, Inc.), Saif Sheikh (Jina 
Pharmaceuticals, Inc.), and Dingjian Liu (Regeneron Pharmaceuticals, Inc.) for their significant contributions in reviewing the article and sharing expert opinions. We also wish to thank Kelly Gerstenberg (AAPS) for helping with the registration and logistic support. Mohd M. Khan is thankful to the AAPS Foundation for a Graduate Student Fellowship Award. Finally, we thank Adam Grobin, Tony Mazzeo, Nanda Subbarao, Kim Huynh-Ba, Jianmei Kochling, and Yan Wu from the AAPS Stability Focus Group for planning the Workshop.

\section{Funding}

Not applicable.

\section{Availability of data and materials}

The meeting agenda and presenters names may be accessible on the AAPS website (https://goo.gl/uBMsQX) and most presentation materials are available on the AAPS Past Presentations website (https://www.aaps.org/ annual-meeting-and-conferences/past-meeting-presentations) although the presentation materials are accessible by password to registered attendees of the workshop.

\section{Authors' contributions}

MMK and BJ made an equal contribution; MMK, BJ, and AM are primary authors of this meeting report, All authors read, commented, and approved the final manuscript.

\section{Competing interests}

All authors declare that they are employees or owners of the companies/ institutions provided and therefore received payments from said companies/ institutions. Otherwise, the authors declare that they have no competing interests.

\section{Publisher's Note}

Springer Nature remains neutral with regard to jurisdictional claims in published maps and institutional affiliations.

\section{Author details}

'University of Maryland, School of Medicine, Baltimore, MD 21201, USA.

${ }^{2}$ University of Maryland, School of Pharmacy, Baltimore, MD 21201, USA

${ }^{3}$ Bristol-Myers Squibb Company, 1 Squibb Drive, New Brunswick, NJ 08903,

USA. ${ }^{4}$ Pharmalytik Consulting \& Training, Newark, Delaware, DE 19711, USA.

Received: 12 October 2017 Accepted: 15 January 2018

Published online: 14 February 2018

\section{References}

Aitchison J (1986) The statistical analysis of compositional data. Chapman and Hall, London, England

Ammann C (2011) Stability studies needed to define the handling and transport conditions of sensitive pharmaceutical or biotechnological products. AAPS PharmSciTech 12(4):1264-1275

Bennett S, McNeil M, Bland L, Arduino M, Villarino M, Perrotta D, Burwen D, Welbel S, Pegues D, Stroud L, Zeitz P, Jarvis W (1995) Postoperative infections traced to contamination of an intravenous anesthetic, propofol. N Engl J Med 333(3):147-154

Capen R, Christopher D, Forenzo P, Ireland C, Liu O, Lyapustina S, O'Neill J, Patterson N, Quinlan M, Sandell D, Schwenke J, Stroup W, Tougas T (2012) On the shelf life of pharmaceutical products. AAPS PharmSciTech 13(3):911-918

EMA (European Agency for the Evaluation of Medicinal Products), CPMP, "Note for Guidance on In-use Stability Testing of Human Medicinal Products" (2001), CPMP/QWP/2934/99

Li H, Nadig D, Kuzmission A, Riley C (2016) Prediction of the changes in drug dissolution from an immediate-release tablet containing two active pharmaceutical ingredients using an accelerated stability assessment program (ASAPprime ${ }^{\circ}$. AAPS Open 2:7

Maier M (2014) DirichletReg: Dirichlet regression for compositional data in R. Research Report Series. Department of Statistics and Mathematics, 125. WU Vienna University of Economics and Business, Vienna Available from http:// epub.wu.ac.at/4077/

Mattner F, Gastmeier P (2004) Bacterial contamination of multiple-dose vials: a prevalence study. Am J Infect Control 32(1):12-16

Muthukumarana S, Tiwari R (2016) Meta-analysis using Dirichlet process. Stat Methods Med Res 25(1):352-365
Nogler-Semenitz E, Lass-Flörl C, Nogler M, Speer G, Dierich M (2007) Bacterial contamination of solutions for Parenteral Administration for Singleand MultipleDose Vials after multiple use in the hospital. Wien Med Wochenschr 157:398-401

PDA Technical Report No. 53 (2012). Guidance for industry: stability testing to support distribution of new drug products. https://store.pda.org/ TableOfContents/TR53_TOC.pdf

Product Quality and Research Institute (PQRI) Stability Shelf Life Working Group. 2007. http://pqri.org/stability-shelf-life-working-group/. Accessed Dec 2017.

Sreedhara A, Yin J, Joyce M, Lau K, Wecksler A, Deperalta G, Yi L, John Y, Kabakoff B, Kishore R (2016) Effect of ambient light on lgG1 monoclonal antibodies during drug product processing and development. Eur J Pharm Biopharm 100:38-46

Stroup W, Quinlan M (2016) Statistical considerations for stability and the estimation of shelf life. In: Zhang L (ed) Nonclinical statistics for pharmaceutical and biotechnology industries. Statistics for biology and health. Springer, Cham

The United States Pharmacopeia (USP) General Chapter Prospectus (1079.1): Storage and transportation of investigational drug products (IDPs). 2016. http://www.uspnf.com/notices/general-chapter-prospectus-storage-andtransportation-investigational-drug-products. Accessed Dec 2017.

Waterman K (2009) Understanding and predicting pharmaceutical product shelflife. In: Huynh-Ba K (ed) Handbook of stability testing in pharmaceutical development. Springer, New York, NY

Waterman K, Chen L, Waterman P, MacDonald B, Monahan A, Scrivens G (2016) Modeling of in-use stability for tablets and powders in bottles. Drug Dev Ind Pharm 42(10):1571-1578

Zhang L (ed) (2016) Nonclinical statistics for pharmaceutical and biotechnology industries. Springer, Heidelberg

\section{Submit your manuscript to a SpringerOpen ${ }^{\circ}$ journal and benefit from:}

- Convenient online submission

- Rigorous peer review

- Open access: articles freely available online

- High visibility within the field

- Retaining the copyright to your article

Submit your next manuscript at $>$ springeropen.com 